\title{
Functional Rejuvenation of Recurrent Laryngeal Nerve after Excision of Parathyroid Cyst: A Case Report and Systematic Review of Literature
}

\author{
N Vishnu S Reddy ${ }^{1}$, Manoj Sharma ${ }^{2}$, Murali Chintham ${ }^{3}$, Priyanka Sharma ${ }^{4}$
}

\begin{abstract}
Vocal fold paralysis is a prevalent manifestation of various disorders inclusive of the commonest conditions, such as, cardiothoracic surgeries, head and neck malignancies, whereas parathyroid cysts and cervical osteophytes are the rarest causes of this common presentation.

A comprehensive systematic review of the literature was done with keyword "parathyroid cysts causing vocal fold palsy" using MEDLINE, PubMed, additionally following PRISMA 2009 guidelines.

A 59-year-old male patient presented with hoarseness of voice with right-sided neck swelling for one year. Excision of swelling adherent to the carotid sheath was done. Postoperative histopathological and biochemical analysis of aspirated fluid was consistent with the parathyroid cyst.

Patient is in regular follow-up, and there are no clinical signs of recurrence and there is normal functioning of the affected vocal cord. This report adds to the growing knowledge of rare parathyroid cysts with unusual presentations, along with an understanding of pathophysiology, clinical diagnosis, and recovery options for vocal fold palsy due to lesions in the parathyroid gland.
\end{abstract}

Keywords: Carotid sheath, Hoarseness of voice, Neck cyst, Parathyroid cyst, Vocal cord palsy, Whole parathyroid hormone (wPTH) assays. Otorhinolaryngology Clinics: An International Journal (2020): 10.5005/jp-journals-10003-1351

\section{INTRODUCTION}

A vocal fold paresis or paralysis represents a diagnostic challenge for the clinician due to complex anatomy and course of innervating fibers especially on the left side; thus, the evaluation and follow-up of such patients should not be limited to routine differentials. Hoarseness of the voice is a common presentation of recurrent laryngeal nerve involvement due to any cause and can be a symptom of serious disease. Resenthal et al. ${ }^{1}$ in 2007 concluded that out of 827 patients suffering from vocal fold immobility, 37\% were due to surgical procedures. The etiological trend has changed such that there has been a shift from extralaryngeal malignancies to nonthyroid surgical procedures as a major cause (66\%). The etiology of unilateral recurrent laryngeal nerve paralysis must be thoroughly investigated.

Benign parathyroid cysts are extremely rare causes of vocal fold paralysis and should be considered as part of the differential diagnosis, in cases of vocal fold paralysis associated with neck swelling. Parathyroid cysts in either the neck or mediastinum are extremely uncommon. The incidence has been stated to vary from $0.08 \%$ as reported by Welti and Gerard-Marchant to $2.8 \%$ as identified by Gilmour, ${ }^{2}$ with only 300 cases reported in the literature. Parathyroid cysts affect both males and females with preponderance in females with a proportion of 2.5:1 women to men. ${ }^{3}$ The location of parathyroid cysts has been described from the angle of mandible up to the superior mediastinum, with dominance on the left side. Cysts of parathyroid origin are conventionally classified as either nonfunctional or functional cysts. Parathyroid cysts are customarily asymptomatic entities of the neck and mediastinum; in contrast, symptomatic parathyroid cysts are extremely rare in occurrence. Ordinarily asymptomatic clinical entity sometimes got incidentally diagnosed during clinical workups for thyroid disorders or other neck and chest soft tissue
${ }^{1-3}$ Department of ENT, Care Hospital, Hyderabad, Telangana, India

${ }^{4}$ Department of ENT, Government District Hospitals, Lakhimpur Kheri, Uttar Pradesh, India

Corresponding Author: Manoj Sharma, Department of ENT, Care Hospitals, Hyderabad, Telangana, India, Phone: +91 9936868509, e-mail: dr.sharmamanoj@yahoo.com

How to cite this article: Reddy NVS, Sharma M, Chintham $M$, et al. Functional Rejuvenation of Recurrent Laryngeal Nerve after Excision of Parathyroid Cyst: A Case Report and Systematic Review of Literature. Int J Otorhinolaryngol Clin 2020;12(2):31-34.

Source of support: Nil

Conflict of interest: None

tumors. The clinical presentation and diagnosis are consistent with symptoms of hyperparathyroidism and hypercalcemia in functional cysts and on histopathology after excision in nonfunctional cysts. A few studies on vocal fold palsy demonstrated parathyroid cysts as a rare causative factor for compression on the recurrent laryngeal nerve within the neck and superior mediastinum.

\section{Case Description}

A 59-year-old male patient presented with hoarseness of voice for one year along with a swelling in the right side of the neck. Past history suggests needle aspiration 6 months ago with the disappearance of swelling without improvement in the quality of voice. Further, there was a gradual recurrence of swelling within 8-10 weeks.

Examination of swelling revealed a large smooth lesion measuring about $80 \times 50 \mathrm{~mm}^{2}$ identified in the right anterior triangle of neck anterior to anterior border of the sternocleidomastoid 


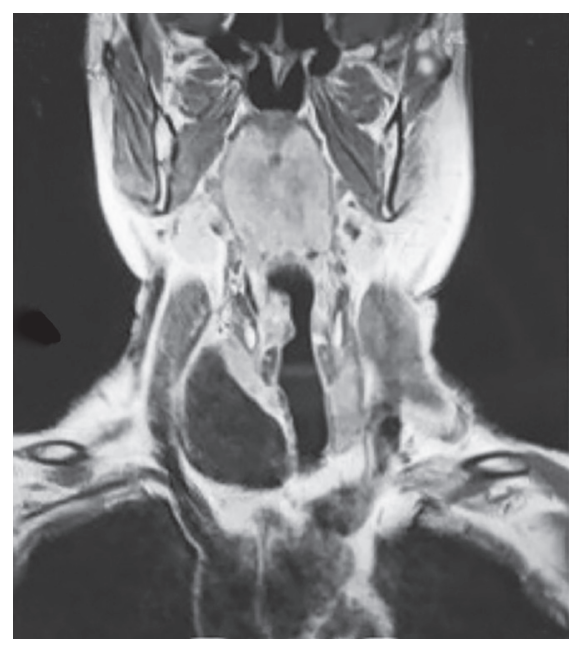

Fig. 1: T1-weighted MRI scan showing large cyst occupying tracheoesophageal groove on the right side

muscle in the lower one-third region. On palpation, the swelling was tense, smooth surfaced, horizontally mobile, nontender with transmitted pulsations from the carotid artery without local rise of temperature and not associated with any significant lymphadenopathy.

Flexible nasopharyngolaryngoscopic examination demonstrated right vocal fold palsy without any local lesion and partially compensated by corresponding left vocal fold. Laboratory evaluation was unremarkable in conjunction with normal thyroidstimulating hormone (TSH) and serum calcium levels. Computerized tomography and contrast magnetic resonance imaging (MRI) scan demonstrated cystic lesion of size $83 \times 56 \times 45 \mathrm{~mm}^{3}$ filled with fluid, attached to the carotid sheath (Fig. 1). Fine needle aspiration cytology (FNAC) revealed the cystic nature of the swelling with inconclusive diagnosis.

Surgical excision and biopsy of the lesion were planned with differential diagnosis of thyroid cyst, branchial cyst, lymphatic swelling, and vascular lesion. Intraoperative lesion was pulsating thick walled and firmly adherent to the anterior wall of the right carotid sheath. In order to scale down the volume of the lesion, $10 \mathrm{~mL}$ amber colored fluid was aspirated from the cyst and sent for biochemical analysis (Fig. 2). Thorough exploration and excision from the carotid sheath were done without violating the integrity of the cyst wall (Fig. 3). Excised sample and aspirated fluid were sent for histopathological and biochemical analysis, respectively. The postoperative course was uneventful, and the patient was discharged from the hospital on the second postoperative day.

\section{Histopathology}

Collapsed cyst lined by cuboidal to low columnar epithelial cells surrounded by islands of chief cells was suggestive of the parathyroid cyst. Analysis of aspirated fluid revealed few epithelial cells. Biochemical analysis of the aspirated fluid revealed PTH (wPTH) level of $564 \mathrm{pg} / \mathrm{mL}$ with normal postoperative serum PTH (53 pg/mL) and serum calcium $(9.8 \mathrm{mg} / \mathrm{dL}$ ) levels.

After two months of follow-up, the patient showed complete recovery of recurrent laryngeal nerve function and symptomatic recovery with normal voice quality. After one-year follow-up, there is no clinical evidence of recurrence of swelling or variation in serum parathyroid hormone and serum calcium levels.

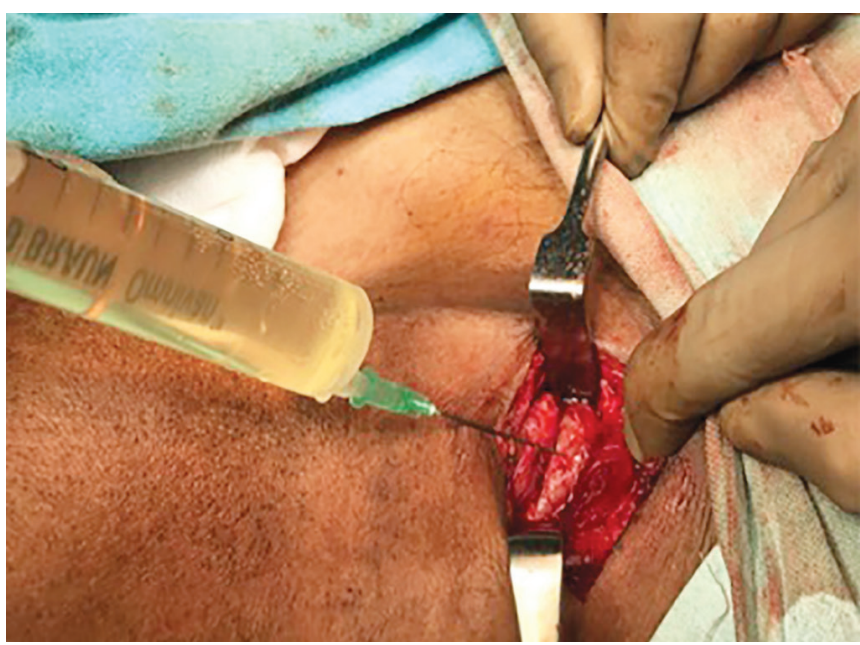

Fig. 2: Aspiration of cyst fluid in order to reduce the volume of swelling for precise dissection from the carotid sheath

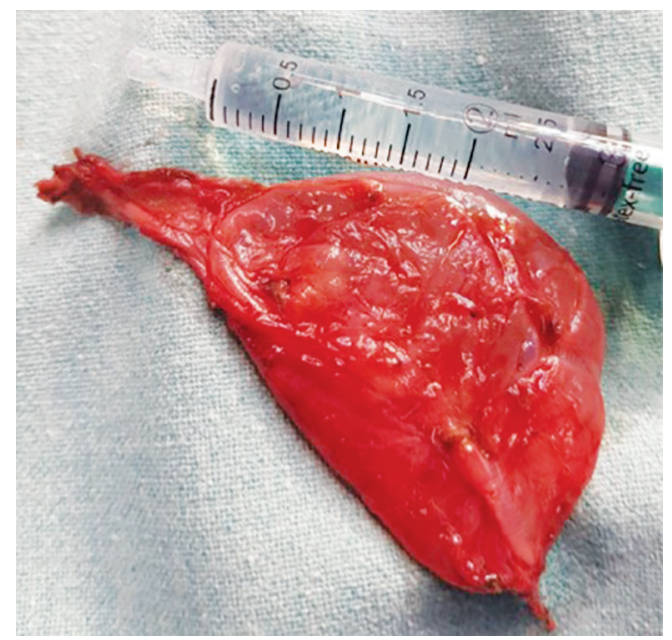

Fig. 3: Excised sample of the parathyroid cyst from the right side of the neck

\section{Discussion}

\section{Surgical History of the Parathyroid Gland}

The parathyroid gland is the last mammalian organ discovered to be visible with the naked eye by Sir Richard Owen in 1849 during an autopsy on an Indian rhinoceros. In 1880, Ivar Sandstorm defined these parathyroid bodies as histologically definable separate entities. Sir John Bland-Sutton did the first intentional surgery on the parathyroid gland in 1909 in 38-year-old patient with a clinical diagnosis of the parathyroid cyst. ${ }^{4}$ In 1953, Cril G Jr${ }^{5}$ reported the first-ever case of parathyroid cyst presented as vocal fold palsy situated in the mediastinum, and almost four decades later, Coates ${ }^{6}$ described the first case of vocal fold palsy caused by parathyroid cyst situated in the neck.

The case reported here is the eleventh case of parathyroid cyst in the neck and third case of the right side neck involvement causing compression of ipsilateral recurrent laryngeal nerve in the tracheoesophageal groove leading to vocal fold palsy, treated with surgical excision and post-op complete functional recovery of the right recurrent laryngeal nerve (Table 1). 
Parathyroid Cyst as a Cause of Vocal Cord Palsy

Table 1: All reported* cases of the parathyroid cysts in the neck presented with RLN palsy

\begin{tabular}{|c|c|c|c|c|c|c|c|}
\hline Serial no. & Sex & Year of publication & Age of presentation & Size of cyst $(\mathrm{mm})$ & Side of cyst & Management & Post-op RLN function \\
\hline 1 & $M$ & 1991 & 60 & 80 & Left & Aspiration & Paralysis $^{6}$ \\
\hline 2 & $M$ & 2006 & 49 & 48 & Right & Surgical & Normal $^{16}$ \\
\hline 3 & $M$ & 2008 & 83 & 39 & Left & Surgical & Normal ${ }^{17}$ \\
\hline 4 & M & 2015 & 30 & 30 & Left & Surgical & Normal $^{18}$ \\
\hline 5 & $\mathrm{~F}$ & 1993 & 36 & 25 & Left & Surgical & Normal $^{19}$ \\
\hline 6 & $\mathrm{~F}$ & 2000 & 37 & 25 & Left & Aspiration & Normal $^{12}$ \\
\hline 7 & $\mathrm{~F}$ & 2011 & 50 & 42 & Left & Aspiration & Normal $^{20}$ \\
\hline 8 & $\mathrm{~F}$ & 2012 & 58 & 32 & Right & Surgical & Normal $^{21}$ \\
\hline 9 & $\mathrm{~F}$ & 2017 & 76 & 22 & Left & Surgical & Paralysis ${ }^{22}$ \\
\hline 10 & $\mathrm{~F}$ & 2017 & 16 & 40 & Left & Surgical & Normal $^{23}$ \\
\hline
\end{tabular}

Excluding present case, *All neck parathyroid cysts associated with vocal cord palsy reported till date are nonfunctional except this case; RLN, recurrent laryngeal nerve

\section{Literature Review}

In a study done by Rowe-Jones et al., out of 2408 patients, 29 patients had a preoperative vocal cord palsy and 22 patients were associated with benign thyroid disease. Return of cord movement after surgery occurred in $89 \%$ of the patients with a benign compressive disease of the thyroid gland?.

In 2014, Ceulemans et al. ${ }^{8}$ reported a rare case of right recurrent laryngeal nerve palsy caused by ipsilateral tracheal diverticulum, whereas surgical resection of the lesion demonstrated complete recovery of nerve function. Unilateral vocal fold palsy rarely can be seen in patients with cervical osteophytes ${ }^{9}$ (only two cases reported) and benign parathyroid cyst causing compression on recurrent laryngeal nerve at any locus within entire course of nerve fibers in the neck and superior mediastinum.

Miazaki et al. ${ }^{10}$ in 2016 reported that three cases of benign thyroid disease presented with vocal cord palsy got completely recovered after surgical excision of gland.

Thorough and systemic review of the literature revealed the parathyroid cyst as a rare entity, and the effect of this disease on vocal folds is extremely rare with only 20 cases reported till date. Electronic review of all published reports of parathyroid cyst causing vocal fold palsy revealed that in 10 cases (50\%) cyst was located in the mediastinum and in 10 cases (50\%) positioned in the neck (excluding patient reported here). Females are more commonly affected in the fourth to sixth decades of life with a slight preponderance on the left side (Table 1). This is the third case of parathyroid cyst situated in the neck with total postoperative recovery of recurrent laryngeal nerve palsy after surgical excision.

\section{Pathophysiology}

Nonfunctioning cysts of parathyroid origin are true cysts derived as developmental ontogenic formations, arising from the $3 \mathrm{rd}$ and 4th branchial clefts. ${ }^{11}$ It has been hypothesized that the mechanism of cyst formation is the coalescence of microcysts into macrocysts. Gradual local degeneration of gland or retention of colloid secretion may be responsible for the formation of microcysts. ${ }^{12}$ Any anatomical compression ranging from medulla oblongata up to vagus nerve or recurrent laryngeal nerve all can cause vocal fold palsy. Left side RLN course is longer and more strenuous than the right. Nonthyroid surgeries are the commonest causes for left recurrent laryngeal nerve involvement due to its lengthy course within the neck and mediastinum. Paralysis of the right vocal fold should be investigated more judiciously as nerve on the right side is shorter and mostly involved by conditions in the neck only. In light of this, complexity decisive diagnosis of vocal fold paralysis requires full realization of the relevant neural anatomy in conjunction with adjacent framework. Subsequently, there are an endless number of vocal fold palsy etiologies, inclusive of neurological, neoplastic, systemic (e.g., sarcoid, lupus, and amyloid), pharmacological (e.g., the dose-dependent neurotoxicity of vinca alkaloids), traumatic, iatrogenic (e.g., thyroid surgery, intubation, anterior cervical approaches, and carotid endarterectomy), and idiopathic.

\section{Clinical Diagnosis}

Unilateral vocal fold palsy causes incompetent laryngeal closure during cough, swallowing, and phonation and may precipitate dyspnea during exertion. The fall in prephonatory glottal pressure due to air leak is responsible for rough and breathy voice. The diagnosis of parathyroid cyst is difficult and frequently misdiagnosed as thyroid pathology; particularly, nonfunctional cysts are often considered as thyroid cysts. Parathyroid hormone levels and thyroid hormone assessment in aspirated fluid can determine whether the cyst is of parathyroid or thyroid origin, but cannot differentiate between functional and nonfunctional parathyroid cysts since only serum PTH and serum calcium levels can reveal the difference. Diagnosis of functional cysts is done either by elevated serum calcium levels with elevated serum parathyroid hormone along with the detection of PTH in aspirated fluid. Evaluation of the intracystic level of PTH is more important than the appearance of the fluid. PTH levels are increased in all parathyroid cysts independently of whether they are functioning or nonfunctioning. ${ }^{13}$ The final diagnosis of parathyroid cysts can only be made with the detection of parathyroid hormone in cystic fluid and histopathological confirmation. Aspirated fluid may be yellow or brown due to a degenerated or infracted parathyroid adenoma containing hemosiderin with the presence of the parathyroid hormone in the fluid. Computerized tomography, magnetic resonance imaging, and parathyroid scintigraphy are useful in the diagnosis of parathyroid cysts. Ultrasound-guided aspiration and biochemical analysis for PTH hormone are the standard diagnostic procedures for this entity and frequently relieve the pressure symptoms if present. The analytical methods for parathyroid hormone include first-generation radioimmunoassays introduced in 1959, second-generation immunometric assays (1987), and recently introduced in the 
year 2000 third-generation "whole PTH assays." Existence of co-expression of PTH/PTH-related peptide receptor 1 and Gcm-2 gene (glial cells missing gene) in parathyroid cyst may serve as an accessory predictive marker for the diagnosis and pathogenesis of disease. $^{14}$

\section{Treatment}

Treatment options include ultrasound-guided aspiration and surgical excision of large cysts causing compressive symptoms. Sclerotherapy has been used but is not universally recommended because of complications such as neurotoxicity and recurrent laryngeal nerve palsy due to aseptic inflammation. ${ }^{15}$ Uncomplicated nonfunctional parathyroid cysts may be treated by aspiration under ultrasound guidance. Surgical excision should be recommended in patients with complications such as recurrent laryngeal nerve palsy, dysphagia, and airway compression. For functional cysts, surgery is always indicated. Hypocalcemia has been reported following the removal of functional cysts despite the normal morphology of other glands; therefore, all cases should be monitored for serum calcium levels in the immediate post-op period and treated appropriately with calcium and vitamin $\mathrm{D}$.

\section{CONCLUSION}

This is the third case of the parathyroid cyst, right side in the neck, with total postoperative recovery of right recurrent laryngeal nerve function, after surgical excision.

Lower one-third and anterior to sternocleidomastoid swellings should be suspected for the parathyroid cyst in addition to thyroid cyst and branchial cyst.

Early and complete surgical excision is always the best treatment when compared to aspiration of cystic fluid to relieve the compressive symptoms on recurrent laryngeal nerve.

Adherence to the surrounding vital structures should be kept in mind during surgery as in this case cyst was firmly adhered to the anterior wall of the right carotid sheath.

Serum calcium, phosphate, alkaline phosphatase, and parathyroid hormone levels should be assessed in every case of the functional parathyroid cyst.

Future research should include the search for further biomarkers for diagnosis and for follow-up of patients with the functional parathyroid cyst.

\section{References}

1. Rosenthal LH, Benninger MS, Deeb RH. Vocal fold immobility: a longitudinal analysis of etiology over 20 years. Laryngoscope 2007;117:1864-1870. DOI: 10.1097/MLG.0b013e3180de4d49.

2. Shields TW, LoCicero J, Reed CE, et al. General thoracic surgery. Philadelphia: Lippincott Williams \& Wilkins; 21-Dec-2011. page no 2546.

3. Ihm PS, Dray T, Sofferman RA, et al. Parathyroid cysts: diagnosis and management. Laryngoscope 2001;111(9):1576-1578. DOI: 10.1097/00005537-200109000-00015.
4. Delbridge LW, Palazzo FF. First parathyroid surgeon: Sir John BlandSutton and the parathyroids. ANZ J Surg 2007;77(12):1058-1061. DOI: 10.1111/j.1445-2197.2007.04324.x.

5. Crile G Jr, Perryman RG. Parathyroid cysts; report of five cases. Surgery 1953;14:151-154.

6. Coates G, Pearman K, Holl-Allen RT. Recurrent nerve palsy due to parathyroid cyst. Int Surg 1991;76(3):192-193.

7. Rowe-Jones JM, Rosswick RP, Leighton SEJ. Benign thyroid disease and vocal cord palsy. Ann R Coll Surg Engl 1993;75(4):241-244.

8. Ceulemans LJ, Lerut $P$, De Moor $S$, et al. Recurrent laryngeal nerve paralysis by compression from a tracheal diverticulum. Ann Thorac Surg 2014;97(3):1068-1071. DOI: 10.1016/j.athoracsur.2013.06.118.

9. Virk JS, Majithia A, Lingam RK, et al. Cervical osteophytes causing vocal fold paralysis: case report and literature review. J Laryngol Otol 2012;126(9):963-965. DOI: 10.1017/S0022215112001259.

10. Miazaki AP, Araújo-Filho VJ, Brandão LG, et al. Vocal fold mobility alteration reversed after thyroidectomy. Autops Case Rep 2016 Sep 30;6(3):53-57. DOI: 10.4322/acr.2016.039.

11. Marsot-Dupuch K, Levret N, Pharaboz C, et al. Congenital neck masses. Embryonic origin and diagnosis. Report of the CIREOL. J Radiol 1995;76(7):405-415.

12. Ippolito G, Palazzo FF, Sebag F, et al. A single-institution 25-year review of true parathyroid cysts. Langenbecks Arch Surg 2006;391(1):13-18. DOI: 10.1007/s00423-005-0579-y.

13. Guner A, Karyagar S, Ozkan O, et al. Parathyroid cyst: the forgotten diagnosis of a neck mass. J Surg Case Rep 2011;2011(8):4. DOI: 10.1093/ jscr/2011.8.4.

14. Lima AC, Fregnani ER, Silva-Sousa YT, et al. Parathyroid hormone/ parathyroid hormone-related peptide receptor 1 expression in odontogenic cystic lesions. Int Endod J 2012;45(3):209-214. DOI: 10.1111/j.1365-2591.2011.01961.x.

15. Sen P, Flower N, Papesch $M$, et al. A benign parathyroid cyst presenting with hoarse voice. J Laryngol Otol 2000;114(2):147-148. DOI: 10.1258/0022215001904923.

16. Coelho DH, Boey HP. Benign parathyroid cyst causing vocal fold paralysis: a case report and review of the literature. Head Neck 2006;28(6):564-566. DOI: 10.1002/hed.20391.

17. Woo EK, Simo B, Conn B, et al. Vocal fold paralysis secondary to a benign parathyroid cyst: a case report with clinical, imaging and pathological findings. Eur Radiol 2008;18(9):2015-2018. DOI: 10.1007/ s00330-008-0919-8.

18. Arduc A, Tutuncu YA, Dogan BA, et al.. Parathyroid cyst. Am Surg 2015;81:163-165.

19. Grey $A B$, Shaw JH, Anderson NE, et al. Parathyroid cyst with recurrent vocal fold paresis. Aust N Z J Surg 1993;63(7):561-562. DOI: 10.1111/ j.1445-2197.1993.tb00453.x.

20. Ghervan C, Goel P. Parathyroid cyst, a rare cause of cystic cervical lesion. Case report. Med Ultrason 2011;13(2):157-160.

21. Khan $A$, Khan $Y$, Raza S, et al. Functional parathyroid cyst: a rare cause of malignant hypercalcinemia with primary hyperparathyroidism - a case report and review of literature. Case Rep Med 2012;2012: 851941. DOI: $10.1155 / 2012 / 851941$.

22. Fustar Preradovic L, Danic D, Dzodic R. Small nonfunctional parathyroid cysts: single institution experience. Endocr J 2017;64(2):151-156. DOI: 10.1507/endocrj.EJ16-0398.

23. Lall A, Haque I, Kumar R, et al. Vocal cord palsy secondary to parathyroid cyst in the young: rare case report with review of the literature. J Head Neck Physicians Surg 2017 Jul-Dec;5(2):90. DOI: 10.4103/jhnps.jhnps_24_17. 